PROCEEDINGS OF THE

AMERICAN MATHEMATICAL SOCIETY

Volume 127, Number 5, Pages 1323-1329

S 0002-9939(99)04694-8

Article electronically published on February 4, 1999

\title{
SEMI-INVARIANTS AND WEIGHTS OF GROUP ALGEBRAS OF FINITE GROUPS
}

\author{
D. S. PASSMAN AND P. WAUTERS
}

(Communicated by Lance W. Small)

\begin{abstract}
We study the semi-invariants and weights of a group algebra $K[G]$ over a field $K$ of characteristic zero. Specifically, we show that certain basic results which hold when $G$ is a polycyclic-by-finite group with $\Delta^{+}(G)=1$ need not hold in the case of group algebras of finite groups. This turns out to be a purely group theoretic question about the existence of class preserving automorphisms.
\end{abstract}

\section{$\S 1$. INTRODUCTION}

Throughout, $K$ will denote a field of characteristic zero. If $G$ is a group, then $G$ acts on the group algebra $K[G]$ by inner automorphisms. Therefore, following [Sm] for example, a nonzero element $\alpha \in K[G]$ is said to be a semi-invariant of $K[G]$ if $\alpha^{g}=\lambda(g) \alpha$ for all $g \in G$. Here, $\lambda \in \widehat{G}=\operatorname{Hom}\left(G, K^{*}\right)$ is the weight of $\alpha$, and we denote the set of all such weights by $\Lambda(G)=\Lambda(G, K)$. If $\lambda \in \Lambda(G)$, we let $(K[G])_{\lambda}$ be the subspace of $K[G]$ consisting of 0 and all those semi-invariants with weight $\lambda$. Furthermore, the semicentre Sz $K[G]$ is defined to be the subalgebra of $K[G]$ given by $\sum_{\lambda \in \Lambda(G)}(K[G])_{\lambda}=\oplus \sum_{\lambda \in \Lambda(G)}(K[G])_{\lambda}$.

Let $\Delta=\Delta(G)$ denote the F.C.-centre of $G$, that is, the set of all elements of $G$ having only finitely many conjugates. Then $\Delta$ is a characteristic subgroup of $G$ which is known to be torsion-free abelian when $K[G]$ is prime. If $x \in \Delta$, $\lambda \in \operatorname{Hom}\left(G, K^{*}\right)$ and $\mathbb{C}_{G}(x) \subseteq \operatorname{ker} \lambda$, then it follows from [MP1, Lemma 3] that $\alpha(\lambda, x)=\sum_{t \in T} \lambda(t)^{-1} x^{t}$ is a semi-invariant with weight $\lambda$, when $T$ is a right transversal for $\mathbb{C}_{G}(x)$ in $G$. Conversely, if $\beta \in(K[G])_{\lambda}$, then $\operatorname{supp} \beta$ is a union of $G$-conjugacy classes of elements $x \in \Delta(G)$, and $\beta$ is a $K$-linear combination of the elements $\alpha(\lambda, x)$ as constructed above. In particular, if $\lambda \in \Lambda(G)$, then ker $\lambda \supseteq \mathbb{C}_{G}(x) \supseteq \mathbb{C}_{G}(\Delta)$ and hence $\Lambda(G) \subseteq \operatorname{Hom}\left(G / \mathbb{C}_{G}(\Delta), K^{*}\right)$.

Now, if $G$ is polycyclic-by-finite and $K[G]$ is prime, then it follows from [MP2, Lemma 2] that $\mathbb{C}_{G}(\Delta)=\mathbb{C}_{G}(g)$ for some $g \in \Delta(G)$. Consequently, if $\lambda \in$ $\operatorname{Hom}\left(G / \mathbb{C}_{G}(\Delta), K^{*}\right)$, then $\operatorname{ker} \lambda \supseteq \mathbb{C}_{G}(\Delta)=\mathbb{C}_{G}(g)$ and hence $\lambda \in \Lambda(G)$. In other words, as was shown in $[\mathrm{Wu}]$, we have $\Lambda(G)=\operatorname{Hom}\left(G / \mathbb{C}_{G}(\Delta), K^{*}\right)$, and thus

Received by the editors September 2, 1997.

1991 Mathematics Subject Classification. Primary 16S34, 20D15, 20 D45.

The first author's research was supported in part by NSF Grant DMS-9622566. The second author's research was supported by an F.W.O.-grant (Belgium). He wishes to thank the Department of Mathematics of the University of Wisconsin-Madison and, in particular, Donald S. Passman and his wife Marjorie for their warm hospitality. 
$\Lambda(G)$ is a finite abelian group. Furthermore, if $K$ is algebraically closed, then [Sm] proved that $\mathrm{Sz} K[G]$ is equal to $K[\Delta]^{G^{\prime}}$, the fixed ring of $K[\Delta]$ under the conjugation action of the commutator subgroup $G^{\prime}$. More generally, [Wu] showed that, for any field $K$, Sz $K[G]=K[\Delta]^{G_{\Lambda}}$ where $G_{\Lambda}$ is defined to be the intersection of the kernels of all weights. From the above formula for $\Lambda(G)$, we have

Lemma 1.1. Let $G$ be a polycyclic-by-finite group and let $K$ be an algebraically closed field. If $K[G]$ is prime, then $\Lambda(G)=\operatorname{Hom}\left(G / G^{\prime} \mathbb{C}_{G}(\Delta), K^{*}\right) \cong G / G^{\prime} \mathbb{C}_{G}(\Delta)$ and hence $G_{\Lambda}=G^{\prime} \mathbb{C}_{G}(\Delta)$.

Proof. Since any $\lambda \in \operatorname{Hom}\left(G, K^{*}\right)$ has $G^{\prime}$ in its kernel, it is clear that $\Lambda(G)=$ $\operatorname{Hom}\left(G / G^{\prime} \mathbb{C}_{G}(\Delta), K^{*}\right)$. The result now follows from the fact that $G / G^{\prime} \mathbb{C}_{G}(\Delta)$ is a finite abelian group.

This, of course, clarifies the fact that $K[\Delta]^{G_{\Lambda}}=K[\Delta]^{G^{\prime}}$ when $K$ is an algebraically closed field.

In the present paper, we consider similar questions, but with $G$ finite. Of course, here $G=\Delta$, so $\mathbb{C}_{G}(\Delta)=\mathbb{Z}(G)$, and we show that the obvious analogs of many of the above results for prime group algebras fail in this case. Indeed, in our first example, based on a construction of [Wl], we find a group $G$ and a linear character $\lambda \in \widehat{G}$ with $\lambda(\mathbb{Z}(G))=1$ such that ker $\lambda$ does not contain the centralizer of any $x \in G$. In particular, $\Lambda(G) \neq \operatorname{Hom}\left(G / G^{\prime} \mathbb{C}_{G}(\Delta), K^{*}\right)$ and we show, in fact, that $\Lambda(G)$ is not even a subgroup of $\widehat{G}$. Our second example is based on a construction of [Sh] and shows that the equality $G_{\Lambda}=G^{\prime} \mathbb{C}_{G}(\Delta)=G^{\prime} \mathbb{Z}(G)$ need not hold in this finite context, even when $K$ is algebraically closed.

We close this section with some observations on the semicentre of the group algebra of a finite group. The first part is, of course, a special case of the result of [Sm] mentioned above.

Lemma 1.2. Let $G$ be finite and let $K$ be an algebraically closed field.

(i) $\mathrm{Sz} K[G]=K[G]^{G^{\prime}}$. In particular, this algebra has a $K$-basis consisting of the sums of the $G^{\prime}$-conjugacy classes of elements of $G$, and hence its $K$-dimension is equal to the number of such classes.

(ii) $\operatorname{dim}_{K} \operatorname{Sz} K[G]=\sum_{x \in \mathcal{X}}\left|G: G^{\prime} \mathbb{C}_{G}(x)\right|$ and

$$
G_{\Lambda}=\bigcap_{x \in \mathcal{X}} G^{\prime} \mathbb{C}_{G}(x)=\bigcap_{x \in G} G^{\prime} \mathbb{C}_{G}(x),
$$

where $\mathcal{X}$ is a set of representatives of the $G$-conjugacy classes of $G$.

Proof. (i) The conjugation action of $G$ on $V=K[G]$ makes $V$ into a $K[G]$-module. Since $K$ is algebraically closed, it follows that $\mathrm{Sz} K[G]$ is precisely the sum of all 1-dimensional submodules of $V$, and this is certainly the set of fixed points under the action of $G^{\prime}$. Finally, since $V$ is a permutation module for $G^{\prime}$, the structure of the $G^{\prime}$-fixed points of $V$ is clear.

(ii) It follows from our previous discussion that $\mathrm{Sz} K[G]$ is the $K$-linear sum of the semi-invariants $\alpha(\lambda, x)$, where $x$ runs through the set $\mathcal{X}$ of representatives of the conjugacy classes of $G$ and where $\lambda \in \operatorname{Hom}\left(G, K^{*}\right)$ with $\operatorname{ker} \lambda \supseteq \mathbb{C}_{G}(x)$. Now, for fixed $x$, the $\lambda \mathrm{s}$ here correspond to all the linear characters of the abelian group $G / G^{\prime} \mathbb{C}_{G}(x)$. Hence there are $\left|G: G^{\prime} \mathbb{C}_{G}(x)\right|$ such semi-invariants, and they are $K$-linearly independent since they have distinct weights. On the other hand, the semi-invariants which correspond to different $G$-conjugacy classes are certainly 
linearly independent in $K[G]$ since they have disjoint supports. Thus the set of all such $\alpha(\lambda, x)$ is a basis for $\mathrm{Sz} K[G]$, and the dimension of the semicentre is the number of these basis elements, namely $\sum_{x \in \mathcal{X}}\left|G: G^{\prime} \mathbb{C}_{G}(x)\right|$. Similarly, the first expression for $G_{\Lambda}$ follows from the fact that, for each $x \in \mathcal{X}$, the intersection of the kernels of all linear characters in $\operatorname{Hom}\left(G / G^{\prime} \mathbb{C}_{G}(x), K^{*}\right)$ is the identity group. Hence, the intersection of the kernels of all $\lambda \in \operatorname{Hom}\left(G, K^{*}\right)$ with $\operatorname{ker} \lambda \supseteq G^{\prime} \mathbb{C}_{G}(x)$ is precisely equal to $G^{\prime} \mathbb{C}_{G}(x)$. Finally, if $x$ and $y$ are conjugate in $G$, then it is clear that $G^{\prime} \mathbb{C}_{G}(x)=G^{\prime} \mathbb{C}_{G}(y)$, and this yields the second expression.

\section{§2. The First EXAmple}

The finite groups we will use in both examples are constructed as semidirect products $G=H \rtimes C$ where $C$ is cyclic and where the map $C \rightarrow \operatorname{Aut}(H)$ has rather special properties.

Lemma 2.1. Let $H$ be a finite group and let $\theta$ be an automorphism of $H$ of order $m>1$ which preserves the conjugacy classes of $H$. Set $G=H \rtimes C$ where $C=\langle c\rangle$ is cyclic of order $m$ and where $c$ acts on $H$ via the automorphism $\theta$. Then

(i) $G^{\prime}=H^{\prime}$ and $\mathbb{Z}(H) \subseteq \mathbb{Z}(G)$.

(ii) If $\lambda \in \operatorname{Hom}\left(G, K^{*}\right)$ with $\operatorname{ker} \lambda=H$, then $\lambda \notin \Lambda(G)$.

(iii) $\mathbb{Z}(G)=\mathbb{Z}(H)$ if no nontrivial power of $\theta$ is inner on $H$.

Proof. (i) Note that $H^{\prime} \triangleleft G$ and that $H / H^{\prime}$ is abelian. Now $\theta$ preserves the classes of $H$, so it also preserves the classes of $H / H^{\prime}$, and hence it must act trivially on this abelian group. It follows that $G / H^{\prime}$ is abelian, and consequently, $G^{\prime} \subseteq H^{\prime}$. Similarly, since $\theta$ preserves the classes of $H$, it acts trivially on $\mathbb{Z}(H)$, and hence $\mathbb{Z}(H) \subseteq \mathbb{Z}(G)$.

(ii) We know that $\lambda \in \Lambda(G)$ if and only if there exists $x \in G$ with $\mathbb{C}_{G}(x) \subseteq$ $\operatorname{ker} \lambda=H$. Consequently, it suffices to show that no such element exists. To this end, suppose $x \in G$ with $\mathbb{C}_{G}(x) \subseteq H$. Then $x \in H$ and, since $\theta$ preserves the classes of $H$, we have $x^{c}=x^{\theta}=x^{h}$ for some $h \in H$. Thus $c h^{-1} \in \mathbb{C}_{G}(x) \subseteq H$ and this yields $c \in H$, a contradiction.

(iii) Finally, if $z \in \mathbb{Z}(G) \subseteq H \rtimes C$, then $z=h^{-1} c^{j}$ for some $h \in H$ and some $j=0,1, \ldots, m-1$. Since $z$ centralizes $H$, it follows that $c^{j}$ and $h$ are equal in their conjugation action on $H$. In particular, $\theta^{j}$ is inner on $H$ and the hypothesis implies that $j=0$ and hence that $z=h^{-1} \in H$. With this, it is clear that $z$ is contained in $\mathbb{Z}(H)$, so $\mathbb{Z}(G) \subseteq \mathbb{Z}(H)$ and part (i) yields the result.

It follows from (ii) and (iii) above that one of the problems discussed in the introduction essentially comes down to finding an example of an outer automorphism which is class preserving. This was first considered by W. Burnside in his book [B]. Here we use a special case of a construction found in [Wl]. Specifically, for the remainder of this section, we fix the following notation.

Let $H=A \rtimes B$ be the semidirect product of $A=\langle a\rangle$, a cyclic group of order 8 , by the fours group $B=\langle x, y\rangle$, where $B$ acts on $A$ by $a^{x}=a^{3}$ and $a^{y}=a^{5}$. Note that $a^{x y}=a^{-1}, \mathbb{C}_{H}(A)=A, \mathbb{Z}(H)=\left\langle a^{4}\right\rangle$ and $H^{\prime}=\left\langle a^{2}\right\rangle$. Furthermore, $x^{a}=x a^{6}, y^{a}=y a^{4}$ and $x^{a^{2}}=x a^{4}$. Next, define $\theta \in \operatorname{Aut}(H)$ by $a^{\theta}=a, x^{\theta}=x a^{4}$, and $y^{\theta}=y a^{4}$. Since $a^{4}$ is central in $H$ and of order 2 , it follows easily that $\theta$ is a well-defined automorphism of $H$ of order 2. Set $G=H \rtimes C$ where $C=\langle c\rangle$ is cyclic of order 2 and where $c$ acts on $H$ via the automorphism $\theta$. Clearly, $|H|=32$ and $|G|=64$. The basic properties of $\theta$ given below are from [Wl]. 
Lemma 2.2. With the above notation, $\theta$ is an outer automorphism of $H$ which preserves the conjugacy classes of the group. In particular, we have $G^{\prime}=H^{\prime}=\left\langle a^{2}\right\rangle$ and $\mathbb{Z}(G)=\mathbb{Z}(H)=\left\langle a^{4}\right\rangle$.

Proof. Since $a^{4}$ is central in $H$, we have $(x y)^{\theta}=x y$. Now suppose, by way of contradiction, that $\theta$ is inner on $H$, say induced by the element $h \in H$. Then, since $a=a^{\theta}=a^{h}$, we have $h \in \mathbb{C}_{H}(A)=A$, so $h=a^{j}$ for some $j=0, \ldots, 7$. From $a^{x y}=a^{-1}$ and $(x y)^{\theta}=x y$, we deduce that $x y=(x y)^{a^{j}}=x y a^{2 j}$. Thus $j=0$ or 4 and $h=a^{j}$ is central in $H$, a contradiction. Next, $\theta$ preserves the conjugacy classes of $H$ since, for all $i$, we have $\left(a^{i}\right)^{\theta}=a^{i},\left(x a^{i}\right)^{\theta}=\left(x a^{i}\right)^{a^{2}},\left(y a^{i}\right)^{\theta}=\left(y a^{i}\right)^{a}$ and $\left(x y a^{i}\right)^{\theta}=x y a^{i}$. The remainder of the result follows from the preceding lemma.

We can now prove

Proposition 2.3. If $G=H \rtimes C$ is as above, then $\Lambda(G)$ is not a subgroup of $\operatorname{Hom}\left(G, K^{*}\right)$, and there exists $\lambda \in \operatorname{Hom}\left(G / G^{\prime} \mathbb{Z}(G), K^{*}\right)$ with $\lambda \notin \Lambda(G)$.

Proof. Since $G^{\prime}=H^{\prime}=\left\langle a^{2}\right\rangle$, it follows that $G / G^{\prime}$ is an elementary abelian 2group of order 16 with generators $a G^{\prime}, x G^{\prime}, y G^{\prime}$ and $c G^{\prime}$. In particular, there exist $\mu, \eta \in \operatorname{Hom}\left(G, K^{*}\right)$ with $\mu(a)=-1, \mu(x)=\mu(y)=\mu(c)=1$ and with $\eta(a)=\eta(c)=-1, \eta(x)=\eta(y)=1$. Now it is easy to check that $\mu, \eta \in \Lambda(G)$. Indeed, these characters are the weights of the semi-invariants $\alpha=x\left(1+a^{4}\right)\left(1-a^{2}\right)$ and $\beta=y\left(1-a^{4}\right)$, respectively. On the other hand, $\lambda=\mu \eta$ satisfies $\lambda(a)=\lambda(x)=$ $\lambda(y)=1, \lambda(c)=-1$, so ker $\lambda=H$. Thus, by Lemmas 2.1(ii) and 2.2, we see that $\mu \eta=\lambda \notin \Lambda(G)$, and consequently $\Lambda(G)$ is not a subgroup of $\operatorname{Hom}\left(G, K^{*}\right)$. Finally, note that $\operatorname{ker} \lambda=H \supseteq\left\langle a^{2}\right\rangle=G^{\prime} \mathbb{Z}(G)$.

Some aspects of this result can be found in [I]. We remark that, in the above proof, $\alpha \beta=0$ since otherwise $\alpha \beta$ would be a semi-invariant with weight $\lambda=\mu \eta$. Furthermore, since $G^{\prime}=\left\langle a^{2}\right\rangle$ and $\mathbb{Z}(G)=\left\langle a^{4}\right\rangle$, we can use Lemma 1.2(i) to quickly describe $\mathrm{Sz} K[G]$. Indeed, let $T=\mathbb{C}_{G}\left(G^{\prime}\right)=\langle a, y, c\rangle$ and note that $G^{\prime} / \mathbb{Z}(G)$ has order 2 and that $|G: T|=2$. Thus since $g^{a^{2}}=a^{4} g$ for all $g \in G \backslash T$, it follows easily that $\mathrm{Sz} K[G]=K[T]+\left(1+a^{4}\right) K[G]$ and that the dimension of this algebra is $|T|+(|G|-|T|) / 2=3|G| / 4=48$.

Finally, it follows from Lemma $1.2($ ii), by considering the classes of the elements $a, x$ and $y$, that $G_{\Lambda}=G^{\prime} \mathbb{Z}(G)$. As we will see in the next section, this equality does not hold for all finite groups.

\section{$\S 3$. THE SECOND EXAMPLE}

Our goal here is to prove the existence of a finite group $G$ such that, in the notation of Lemma $1.2(\mathrm{ii}), G_{\Lambda}=\bigcap_{x \in G} G^{\prime} \mathbb{C}_{G}(x) \neq G^{\prime} \mathbb{Z}(G)$. For this, we study a particular subgroup of an example constructed in the paper [Sh]. To start with, we need

Lemma 3.1. Let $q$ be a power of the prime number $p$. If $n \geq 3$, then every element of the finite field $\mathrm{GF}\left(q^{n}\right)$ is a sum of $(q+1)$ st powers.

Proof. Let $L$ be the additive span of all elements $x^{q+1}$ with $x \in \operatorname{GF}\left(q^{n}\right)$. Since $L$ is clearly closed under addition and multiplication, it follows that $L$ is a subfield of $\operatorname{GF}\left(q^{n}\right)$. We first show that $\operatorname{GF}(q) \subseteq L$. To this end, let $x \in \operatorname{GF}(q) \subseteq \operatorname{GF}\left(q^{n}\right)$ and note that $x^{q}=x$. Thus $x^{2}=x^{q+1} \in L$. But, every element in the finite field $\operatorname{GF}(q)$ is a sum of two squares, and consequently $\operatorname{GF}(q) \subseteq L$. It now follows that 
$L=\operatorname{GF}\left(q^{m}\right)$ for some integer $m$ dividing $n$. Consider the group homomorphism $\varphi: \operatorname{GF}\left(q^{n}\right)^{*} \rightarrow L^{*}$ given by $x \mapsto x^{q+1}$. Since $\operatorname{ker} \varphi=\left\{x \in \mathrm{GF}\left(q^{n}\right) \mid x^{q+1}=1\right\}$ has at most $q+1$ elements, we have

$$
q^{m}-1=\left|L^{*}\right| \geq\left|\operatorname{GF}\left(q^{n}\right)^{*}\right| /(q+1)=\left(q^{n}-1\right) /(q+1) .
$$

In particular, if we write $m t=n$ for some integer $t \geq 1$, then

$$
q+1 \geq\left(q^{m t}-1\right) /\left(q^{m}-1\right)=1+q^{m}+\cdots+\left(q^{m}\right)^{t-1} .
$$

Now, if $t=2$ in the above, then $1+q \geq 1+q^{m}$. Hence $m=1$ and $n=m t=2$, contradicting our assumption that $n \geq 3$. On the other hand, if $t>2$, then the preceding displayed equation yields $1+q \geq 1+q^{m}+q^{2 m}$, again a contradiction. Thus $t=1$, so $n=m t=m$ and $L=\operatorname{GF}\left(q^{n}\right)$, as required.

For the remainder of this section, we let $p, q$ and $n \geq 3$ be as above. Furthermore, we write $F$ for the finite field $\operatorname{GF}\left(q^{n}\right)$, and we let $\phi$ denote the field automorphism of $F$ given by $x \mapsto x^{q}$. Then $\phi$ has order $n \geq 3$ and the fixed field of $\phi$ is $F^{\phi}=\operatorname{GF}(q)$. Following [Sh], we form the $\operatorname{ring} R=F[X ; \phi] /\left(X^{3}\right)$, where $F[X ; \phi]$ is the skew polynomial ring determined by $\phi$. By a slight abuse of notation, we can write $R=F \oplus F X \oplus F X^{2}$ as a 3-dimensional left $F$-vector space with $X^{3}=0$ and with $X f=f^{\phi} X=f^{q} X$ for all $f \in F$. Since $R X=F X \oplus F X^{2}$ is a nilpotent ideal of $R$, it is clear that $U=1+R X$ is a group of units of $R$ with $|U|=|F|^{2}=q^{2 n}$.

Lemma 3.2. Using the above notation, we have

(i) $\mathbb{Z}(R)=F^{\phi}=\mathrm{GF}(q)$.

(ii) $\mathbb{Z}(U)=1+R X^{2}=1+F X^{2}$.

(iii) $U^{\prime}=[U, U]=1+R X^{2}=1+F X^{2}$.

Proof. (i) Since $n \geq 3$, it is clear that $F$ is self-centralizing in $R$. Thus, since $R$ is generated by $F$ and $X$, we have $\mathbb{Z}(R)=\mathbb{C}_{F}(X)=F^{\phi}=\mathrm{GF}(q)$.

(ii) It follows from $X^{3}=0$ that $1+F X^{2} \subseteq \mathbb{Z}(U)$. Conversely, using the $F$-linear independence of the automorphisms 1 and $\phi$, it is easy to see that the centralizer in $U$ of $1+F X$ is contained in $1+F X^{2}$.

(iii) Let $1+r, 1+s \in U$ and note that $(R X)^{3}=0$. Since $r, s \in R X$, we have

$$
\begin{aligned}
{[1+r, 1+s] } & =\left(1-r+r^{2}\right)\left(1-s+s^{2}\right)(1+r)(1+s) \\
& =1+(r s-s r) \in 1+R X^{2} .
\end{aligned}
$$

Consequently, $U^{\prime}=[U, U]$ is a subgroup of $1+R X^{2}=1+F X^{2}$. For the reverse inclusion, let $t$ be a primitive element of $F$, so that $\mathrm{o}(t)=q^{n}-1$, and let $r=a X$ and $s=a t X$ for some $a \in F$. Then, the above displayed equation yields

$$
[1+r, 1+s]=1+a^{q+1} t\left(t^{q-1}-1\right) X^{2},
$$

and note that $t\left(t^{q-1}-1\right) \neq 0$ since $t \neq 0$ and $\mathrm{o}(t)=q^{n}-1$. Furthermore, if $f, g \in F$, then $\left(1+f X^{2}\right)\left(1+g X^{2}\right)=1+(f+g) X^{2}$. Thus, by multiplying commutators for various elements $a \in F$, we see that $[U, U]$ contains $1+L t\left(t^{q-1}-1\right) X^{2}$ where $L$ is the additive span of all $a^{q+1}$ with $a \in F$. By the previous lemma, $L=F$ and hence $[U, U]$ contains $1+F t\left(t^{q-1}-1\right) X^{2}=1+F X^{2}$.

Again, following [Sh], we consider certain subsets of $\mathrm{M}_{2}(R)$ and, for convenience, we abbreviate the matrix $\left(\begin{array}{ll}a & b \\ 0 & 1\end{array}\right)$ by $((a, b))$. Set $H=((U, R))$ and observe that matrix multiplication translates to the formulas $((a, b))((r, s))=((a r, a s+b))$ and 
$((a, b))^{-1}=\left(\left(a^{-1},-a^{-1} b\right)\right)$. Thus $H$ is a group of order $|U||R|=q^{5 n}$, and basic properties of this group are listed below. As will be apparent, we really only need to know the obvious fact that $H^{\prime}$ contains $\left(\left(U^{\prime}, 0\right)\right)$.

Lemma 3.3. If $H$ is the group described above, then

(i) $\mathbb{Z}(H)=\left(\left(1, R X^{2}\right)\right)$.

(ii) $H^{\prime}=[H, H]=\left(\left(1+R X^{2}, R X\right)\right)$.

Proof. (i) Let $z=((a, b)) \in \mathbb{Z}(H)$. Then $z$ commutes with $((1,1))$ and this yields $a=1$. Furthermore, since $z$ commutes with $((1+X, 0))$, it follows that $X b=0$, and hence that $b \in R X^{2}$. Consequently, $\mathbb{Z}(H) \subseteq\left(\left(1, R X^{2}\right)\right)$, and a direct calculation yields the reverse inclusion.

(ii) By the preceding lemma, $\left(\left(1+R X^{2}, 0\right)\right)=\left(\left(U^{\prime}, 0\right)\right) \subseteq H^{\prime}$. Furthermore, if $b \in R$, then the commutator of $((1+X, 0))^{-1}$ and $((1, b))^{-1}$ is equal to $((1, X b))$. Thus $((1, R X)) \subseteq H^{\prime}$ and therefore

$$
\left(\left(1+R X^{2}, R X\right)\right)=\left(\left(1+R X^{2}, 0\right)\right)((1, R X)) \subseteq H^{\prime} .
$$

For the reverse inclusion, consider the maps $\sigma$ and $\tau$ given by $\sigma:((a, b)) \mapsto a U^{\prime}$ and $\tau:((a, b)) \mapsto b+R X$. Then, it follows easily from the formula for matrix multiplication that $\sigma: H \rightarrow U / U^{\prime}$ and $\tau: H \rightarrow(R / R X)^{+}$are group homomorphisms to abelian groups. Here, of course, $(R / R X)^{+}$indicates the additive group of the ring. Consequently, $H^{\prime} \subseteq \operatorname{ker} \sigma \cap \operatorname{ker} \tau=\left(\left(1+R X^{2}, R X\right)\right)$, as required.

Now let $u=1+X^{2} \in U$ and define $\theta: H \rightarrow H$ by $\theta:((a, b)) \mapsto((a, b u))$. Then, we have the following special case of a result of [Sh]. Note, however, that our choice of $u$ yields the stronger conclusion in part (ii) that $k$ is contained in $H^{\prime}$, and not just in $H$. Furthermore, $k^{\theta}=k$.

Lemma 3.4. Let $u$ and $\theta$ be as above.

(i) $\theta$ is an outer automorphism of $H$ of prime order $p$.

(ii) If $h \in H$, then there exists $k \in H^{\prime}$ with $h^{\theta}=h^{k}$ and $k^{\theta}=k$. In particular, $\theta$ preserves the conjugacy classes of $H$.

Proof. (i) The formula for matrix multiplication implies that $\theta$ is an endomorphism of $H$. Furthermore, $\theta^{i}:((a, b)) \mapsto\left(\left(a, b u^{i}\right)\right)$ so, since $u^{p}=\left(1+X^{2}\right)^{p}=1$, it follows that $\theta$ is an automorphism of order $p$.

Suppose, by way of contradiction, that $\theta$ is the inner automorphism induced by $((c, d))^{-1} \in H$. Then, for all $r \in R$, we have

$$
((1, r u))=((1, r))^{\theta}=((c, d))((1, r))((c, d))^{-1}=((1, c r)) .
$$

Thus $r u=c r$, and $r=1$ yields $c=u$. Consequently $1+X^{2}=u \in \mathbb{Z}(R)$, and this contradicts Lemma 3.2(i).

(ii) Let $h=((a, b)) \in H$ and set $k=\left(\left(1+f X^{2}, 0\right)\right)^{-1}$ for some $f \in F$. Note that $k \in H^{\prime}$, by Lemma 3.3(ii), and clearly $k^{\theta}=k$. Since $1+f X^{2} \in \mathbb{Z}(U)$, by Lemma 3.2(ii), it follows easily that the equation $h^{\theta}=h^{k}$ is equivalent to $b\left(1+X^{2}\right)=b u=\left(1+f X^{2}\right) b$ and hence to $b X^{2}=f X^{2} b$. Furthermore, if we write $b=b_{0}+b_{1} X+b_{2} X^{2}$, with $b_{0}, b_{1}, b_{2} \in F$, then using $X^{3}=0$, the latter equation becomes $b_{0} X^{2}=f X^{2} b_{0}=f b_{0}^{\phi^{2}} X^{2}$. But, obviously, this equation can be solved for $f \in F$. Indeed, if $b_{0}=0$, take $f=0$, and if $b_{0} \neq 0$, take $f=b_{0}\left(b_{0}^{-1}\right)^{\phi^{2}}$. In other words, we have shown that an appropriate element $k$ exists. 
Finally, let $G=H \rtimes C$ be the semidirect product of $H$ by the cyclic group $C=\langle c\rangle$ of order $p$, where $c$ acts on $H$ via the automorphism $\theta$. We can now quickly obtain our main result.

Theorem 3.5. Let $G=H \rtimes C$ be as above. Then

(i) $\mathbb{Z}(G)=\mathbb{Z}(H)$ and $G^{\prime}=H^{\prime}$.

(ii) $c \in \bigcap_{x \in G} G^{\prime} \mathbb{C}_{G}(x)$. In particular, if $K$ is an algebraically closed field, then $c \in G_{\Lambda} \backslash G^{\prime} \mathbb{Z}(G)$.

Proof. (i) Since $\theta$ has prime order, it follows from the preceding lemma that no nontrivial power of this automorphism is inner on $H$. The equalities $\mathbb{Z}(G)=\mathbb{Z}(H)$ and $G^{\prime}=H^{\prime}$ now follow from Lemmas 2.1 and 3.4.

(ii) Let $x$ be an arbitrary element of $G=H C$, and write $x=h c^{i}$ for some $h \in H$ and integer $i$. By the preceding lemma again, there exists $k \in H^{\prime}$ with $h^{\theta}=h^{k}$ and $k^{\theta}=k$. The latter equality shows that $k$ and $c$ commute, and hence we have

$$
x^{c}=\left(h c^{i}\right)^{c}=h^{\theta} c^{i}=h^{k} c^{i}=\left(h c^{i}\right)^{k}=x^{k} .
$$

Thus, $c k^{-1} \in \mathbb{C}_{G}(x)$ and, since $k \in H^{\prime}=G^{\prime}$, we conclude that $c=\left(c k^{-1}\right) k \in$ $\mathbb{C}_{G}(x) G^{\prime}=G^{\prime} \mathbb{C}_{G}(x)$. Lemma 1.2(ii) now implies that $c \in \bigcap_{x \in G} G^{\prime} \mathbb{C}_{G}(x)=G_{\Lambda}$, and certainly $c \notin G^{\prime} \mathbb{Z}(G)$ since $G^{\prime}$ and $\mathbb{Z}(G)$ are both contained in $H$.

Note that $|G|=p|H|=p q^{5 n}$. In particular, if we take $q=p$, then $|G|=p^{5 n+1}$ and the smallest such group has $n=3$ and $|G|=p^{16}$.

\section{REFERENCES}

[B] W. Burnside, Theory of Groups of Finite Order, second edition, Cambridge, 1955. MR 16:1086c

[I] I. M. Isaacs, unpublished note.

[MP1] S. Montgomery and D. S. Passman, X-inner automorphisms of group rings, Houston J. Math. 7 (1981), 395-402. MR 83d:16038

[MP2] — X-inner automorphisms of group rings II, Houston J. Math. 8 (1982), 537-544. MR 84d:16044

[Sh] C. H. Sah, Automorphisms of finite groups, J. Algebra 10 (1968), 47-68. MR 37:5287

[Sm] M. K. Smith, Semi-invariant rings, Comm. Algebra 13 (1985), 1283-1298. MR 86g:16018

[Wl] G. E. Wall, Finite groups with class-preserving outer automorphisms, J. London Math. Soc. 22 (1947), 315-320. MR 10:8g

[Wu $\mathrm{P}$. Wauters, The semicentre of a group algebra (to appear in Proc. Edinburgh Math. Soc.).

Department of Mathematics, University of Wisconsin, Madison, Wisconsin 53706

E-mail address: passman@math.wisc.edu

Department of Mathematics, Limburgs Universitair Centrum, B-3590 Diepenbeek, BELGiUM

E-mail address: pwauters@luc.ac.be 